${ }^{1}$ Dept of Internal Medicine and Endocrinology, VU University Medical Center, Amsterdam, The Netherlands. ${ }^{2} \mathrm{MRC}$ Lifecourse Epidemiology Unit, University of Southampton, Southampton General Hospital, Southampton, ${ }^{3}$ Clinical and Experimental Sciences Academic Unit, Faculty of Medicine, University of Southampton, Southampton, ${ }^{4}$ NIHR Southampton Respiratory Biomedical Research Unit, University of Southampton and University Hospital Southampton NHS Foundation Trust, Southampton, ${ }^{5}$ Human Development and Health Academic Unit, Faculty of Medicine, University of Southampton, Southampton, and ${ }^{6}$ NIHR Musculoskeletal Biomedical Research Unit, University of Oxford, Nuffield Orthopaedic Centre, Oxford, UK. ${ }^{7}$ A list of the Southampton Women's Survey Study Group members and their affiliations can be found in the Acknowledgements section.

Correspondence: N.C. Harvey, MRC Lifecourse Epidemiology Unit, University of Southampton, Southampton General Hospital, Tremona Road, Southampton, SO16 6YD, UK. E-mail: nch@mrc.soton.ac.uk

Received: July 092013 | Accepted after revision: Nov 182013 | First published online: Dec 122013

Support statement: The work within the Southampton Women's Survey has been funded by the Medical Research Council, University of Southampton, British Heart Foundation, Food Standards Agency (contracts N05049 and N05071), National Institute for Health Research (NIHR) Southampton Biomedical Research Centre, NIHR Southampton Respiratory Biomedical Research Unit and University Hospital Southampton NHS Foundation Trust and University of Southampton Research Policy Committee.

Conflict of interest: Disclosures can be found alongside the online version of this article at www.erj.ersjournals.com

Acknowledgements: Southampton Women's Survey Study Group members are as follows. P. Taylor: Osteoporosis Centre, University Hospital NHS Trust, Southampton General Hospital, Southampton, UK; S.M. Robinson: MRC Lifecourse Epidemiology Unit, University of Southampton, Southampton General Hospital; M. Hanson: Institute of Developmental Sciences, University of Southampton, Southampton General Hospital. The authors thank the children and their families who participated in the Southampton Women's Survey and all the research staff and computing staff.

\title{
References
}

1 Abrams SA, Coss-Bu JA, Tiosano D. Vitamin D: effects on childhood health and disease. Nat Rev Endocrinol 2013; 9: $162-170$.

2 Charan J, Goyal JP, Saxena D, et al. Vitamin D for prevention of respiratory tract infections: a systematic review and meta-analysis. J Pharmacol Pharmacother 2012; 3: 300-303.

Weiss ST, Litonjua AA. Vitamin D in asthma and allergy: what next? Eur Respir J 2011; 38: 1255-1257.

4 Hollams EM, Hart PH, Holt BJ, et al. Vitamin D and atopy and asthma phenotypes in children: a longitudinal cohort study. Eur Respir J 2011; 38: 1320-1327.

5 Mulligan ML, Felton SK, Riek AE, et al. Implications of vitamin D deficiency in pregnancy and lactation. Am J Obstet Gynecol 2010; 202: 429.

6 Christesen HT, Elvander C, Lamont RF, et al. The impact of vitamin D in pregnancy on extraskeletal health in children: a systematic review. Acta Obstet Gynecol Scand 2012; 91: 1368-1380.

7 Miyake Y, Sasaki S, Tanaka K, et al. Dairy food, calcium and vitamin D intake in pregnancy, and wheeze and eczema in infants. Eur Respir J 2010; 35: 1228-1234.

8 Pike KC, Inskip HM, Robinson S, et al. Maternal late-pregnancy serum 25-hydroxyvitamin D in relation to childhood wheeze and atopic outcomes. Thorax 2012; 67: 950-956.

9 Morales E, Romieu I, Guerra S, et al. Maternal vitamin D status in pregnancy and risk of lower respiratory tract infections, wheezing, and asthma in offspring. Epidemiology 2012; 23: 64-71.

10 Bäz N, Dargent-Molina P, Wark JD, et al. Cord serum 25-hydroxyvitamin D and risk of early childhood transient wheezing and atopic dermatitis. J Allergy Clin Immunol 2014; 133: 147-153.

11 Inskip HM, Godfrey KM, Robinson SM, et al. Cohort profile: the Southampton Women's Survey. Int J Epidemiol 2006; 35: 42-48.

12 Barros AJ, Hirakata VN. Alternatives for logistic regression in cross-sectional studies: an empirical comparison of models that directly estimate the prevalence ratio. BMC Med Res Methodol 2003; 3: 21.

\section{A specific DAMP profile identifies susceptibility to smoke-induced airway inflammation}

\author{
To the Editor:
}

Chronic obstructive pulmonary disease (COPD) is a major cause of morbidity and mortality, with a worldwide prevalence of $9-10 \%$ [1]. COPD is associated with chronic, neutrophilic inflammation in the lungs, causing destruction of lung parenchyma (emphysema) and/or remodelling of the airways with mucus 
hypersecretion (bronchitis) [2]. Chronic exposure to noxious particles and gases, such as cigarette smoke, is the major risk factor for COPD, while susceptibility to the disease has a strong genetic component [2]. While the activity of the innate immune system increases with disease progression during early stages of COPD, the precise nature of the factors that trigger innate immune responses in COPD is currently unknown. Cell damage and death upon exposure to cigarette smoke in COPD may induce the release of damage associated molecular patterns (DAMPs) [3]. Elevated levels of several prototypic DAMPs, including high-mobility group box (HMGB)1, heat shock proteins (HSPs) and S100A8, have been observed in bronchoalveolar lavage (BAL) fluid, serum and epithelial lining fluid of COPD patients [4-6]. DAMPs activate cells of the innate immune system upon binding to pattern recognition receptors, such as toll-like receptors (TLRs) and receptor for advanced glycation end-products (RAGE). Importantly, the AGER gene, which encodes the RAGE receptor, is a genome-wide association study susceptibility gene for COPD [7].

To date, studies investigating the role of DAMPs in COPD have focused on the analysis of a single DAMP. However, while each individual DAMP triggers specific receptors, the combination of DAMPs released upon cigarette smoke-induced cell death probably determines the overall activation of the innate immune system [8]. Hence, we hypothesise that the signature of DAMPs released upon cigarette smoke exposure is critical in driving the early stages of lung inflammation in COPD, and, as such, constitutes a central component in the susceptibility to the disease. Therefore, we related the cigarette smoke-induced DAMP profile to disease susceptibility to develop neutrophilic airway inflammation in vivo. To this end, we used an experimental model where 30 inbred mouse strains were exposed to cigarette smoke or air as a control $(n=8$ per group), for five consecutive days with two exposures per day and one, three or five cigarettes per exposure (fig. 1a). $2 \mathrm{~h}$ after the final cigarette smoke or air exposure, BAL samples were collected to determine neutrophil counts. These 30 strains displayed the full range, from susceptible to nonsusceptible for cigarette smoke-induced neutrophilic airway inflammation (data not shown). For further analyses we selected four strains, with BALB/cByJ as the most susceptible strain, followed by DBA/1J, C57BL/6J and $\mathrm{C} 58 / \mathrm{J}$, the latter strain showing no induction at all of neutrophils after cigarette smoke exposure (fig. 1b). We determined the DAMP signature associated with susceptibility for cigarette smoke-induced neutrophilic airway inflammation by measuring the BAL levels of a selected panel of six DAMPs consisting of calreticulin (CRT), a calcium-binding chaperone molecule that functions as a DAMP upon exposure on the cell membrane or release from secondary necrotic cells [9]; galectin-3, a member of the $\beta$-galactoside-binding lectin protein family, which can activate leukocytes upon release from secondary necrotic cells; S100A8, a member of the calcium-binding S100 protein family, which acts as a DAMP by binding RAGE and TLR-4 [3]; double-stranded (ds)DNA, which can bind to DNA sensors, including TLR-9 [10]; HSP70, a prototypic HSP capable of binding TLR-2/4; and HMGB1, a nuclear nonhistone chromatin-binding protein, that binds to TLR-2/4 and RAGE [3]. The magnitude of the cigarette smoke-induced release varied widely between individual DAMPs, as well as between strains (fig 1c-h). BAL levels of CRT were increased upon cigarette smoke exposure in the highly susceptible BALB/cByJ strain, while no increase was observed in other strains (fig. 1c). Galectin-3 release was increased in all mouse strains after cigarette smoke exposure, with the strongest significant effects in the two most susceptible strains (fig. 1d). A strong and significant induction of S100A8 release was observed upon cigarette smoke exposure in BALB/cByJ and DBA/1J, while no significant induction was observed in the less susceptible strains (fig. 1e). For dsDNA, a strong induction was observed in BALB/cByJ mice, while a smaller increase was observed in DBA/1J and C57BL/6J mice and no increase was observed in the nonsusceptible C58/J mice (fig. 1f). HSP70 levels were increased in BALB/ cByJ, although the levels in the air-exposed group were relatively low when compared with the intermediately susceptible strains DBA/1J and C57BL/6J (fig. 1g). Finally, a relatively small but significant increase in HMGB1 levels was observed upon cigarette smoke exposure compared to air exposure in BALB/ cByJ and C58/J, but not in DBA/1J and C57BL/6J mice (fig. 1h). Using Spearman's $\rho$, we observed a significant correlation $(\mathrm{p} \leqslant 0.01)$ between the increase in DAMP levels and neutrophil counts upon cigarette smoke exposure for CRT $(\rho=0.4518, p=0.0034)$, galectin-3 $(\rho=0.4415, p=0.0049)$, S100A8 $(\rho=0.4390$, $p=0.0052)$, dsDNA $(\rho=0.4813, p=0.0001)$ and HSP70 $(\rho=0.4276, p=0.0059)$, but not for HMGB1 $(\rho=0.3153, p=0.0475)$. To evaluate the difference in DAMP pattern released upon cigarette smoke exposure in susceptible versus nonsusceptible strains, we plotted the relative increase of all six DAMPs for each mouse strain (fig. 1i). Here, a specific combination of DAMPs, i.e. CRT, galectin-3, S100A8 and dsDNA showed an association of cigarette smoke-induced release with increasing susceptibility for neutrophilic airway inflammation, while the cigarette smoke-induced increase in HMGB1 and HSP70 were not associated with susceptibility.

Together, these data show for the first time that genetic susceptibility to cigarette smoke-induced neutrophilia is significantly associated with a specific profile of DAMPs released into the BAL. From the panel of selected DAMPs it appears that the combination of CRT, galectin-3, S100A8 and dsDNA is a reliable marker for susceptibility to cigarette smoke-induced innate immune activation as measured by 
a)

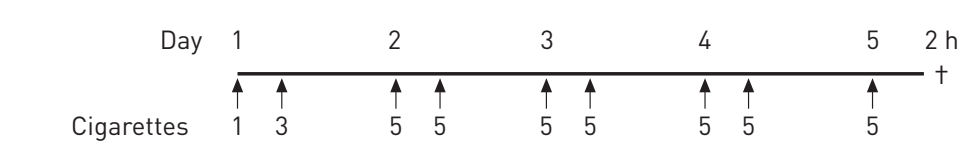
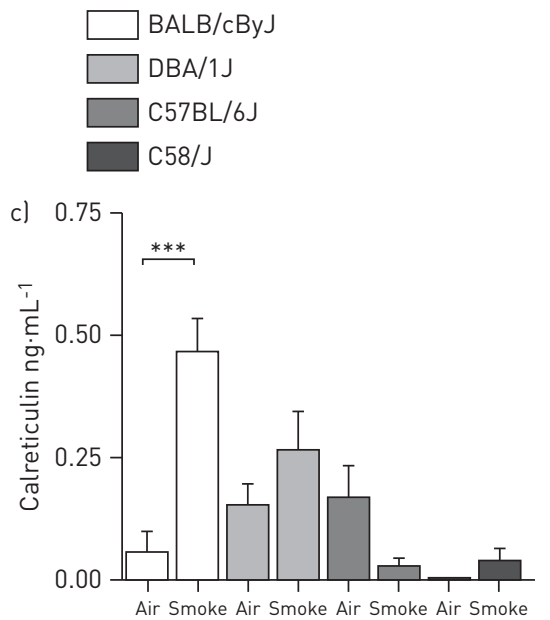

Susceptibility
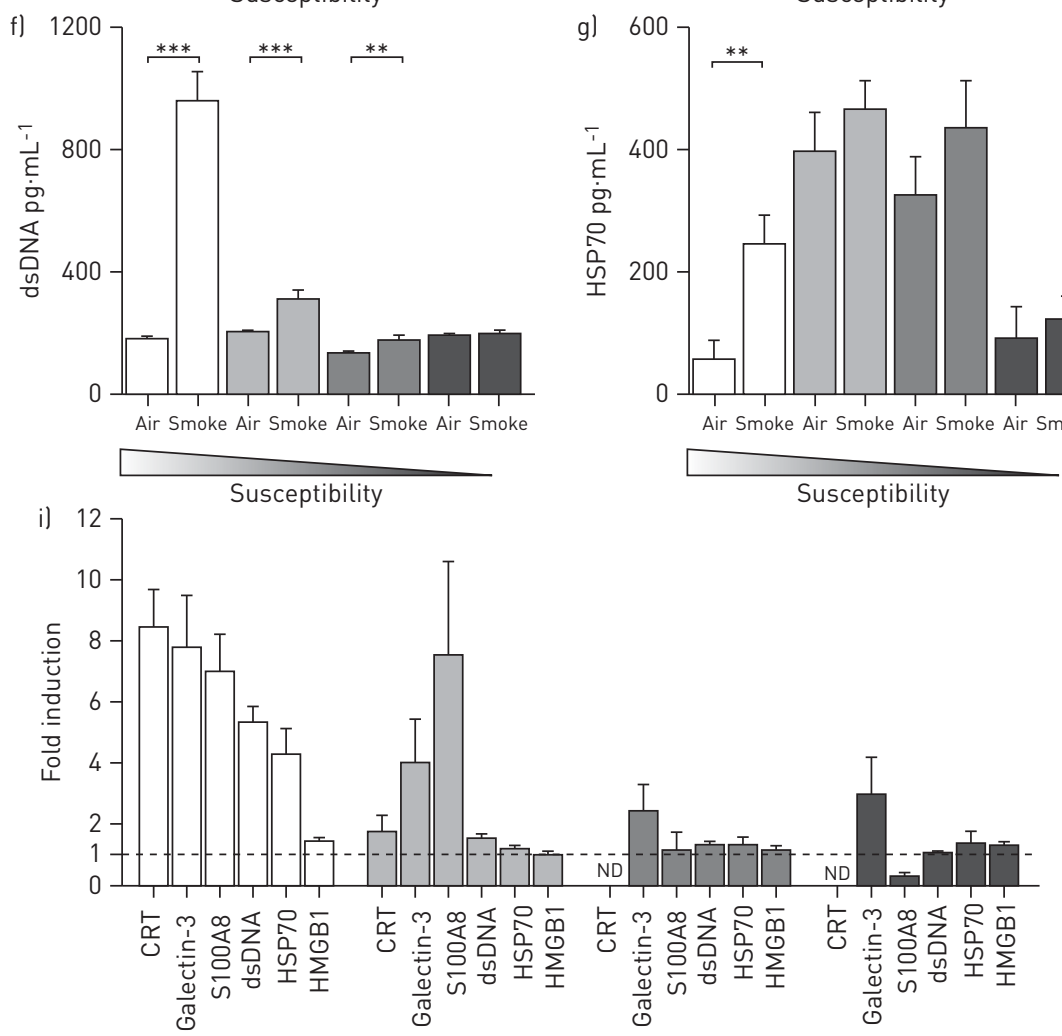
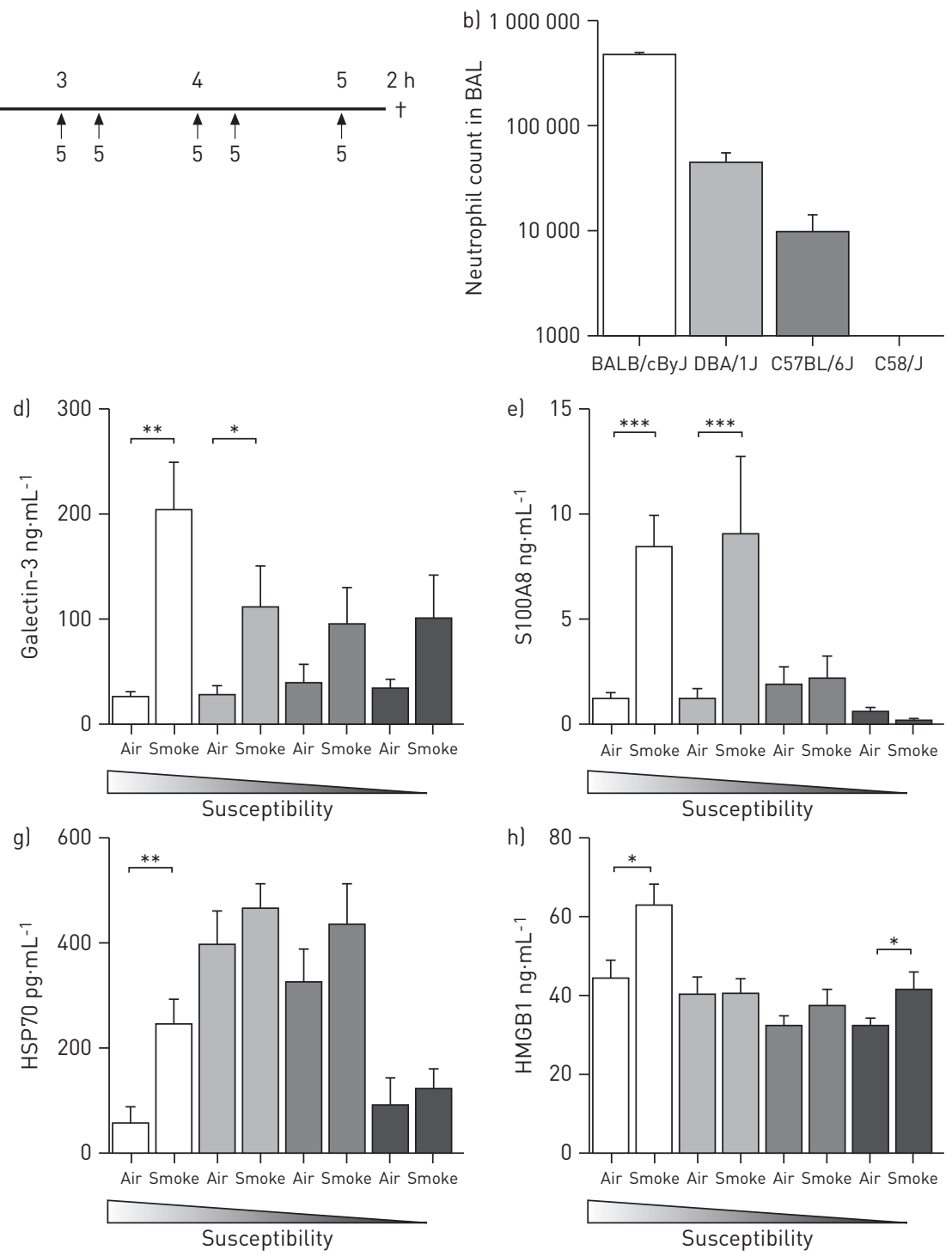

FIGURE 1 Cigarette smoke-induced increase in neutrophil counts and cigarette smoke-induced damage associated molecular pattern (DAMP) release signature in bronchoalveolar lavage (BAL) fluid of susceptible and nonsusceptible mouse strains. a) Schematic representation of the cigarette smoke exposure experiments.

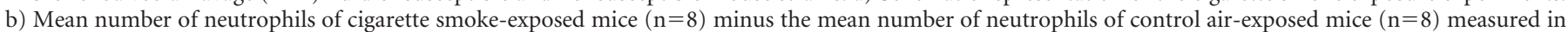
BAL fluid $2 \mathrm{~h}$ after the final cigarette smoke or control air exposure. $\mathrm{c}-\mathrm{h}$ ) Calreticulin (CRT), galectin-3, S100A8, double-stranded (ds)DNA, heat-shock protein

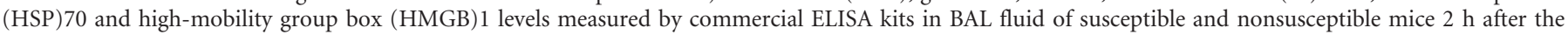
final cigarette smoke exposure $(n=8)$ or control air exposure $(n=8)$. Data are presented as mean \pm SEM. Significance was tested by Mann-Whitney U-test.

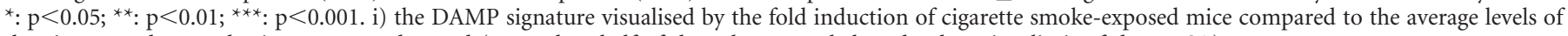
the air-exposed control mice. ND: not detected (more than half of the values were below the detection limit of the ELISA). 
neutrophilic airway inflammation. Our data indicate that the increase of a specific profile of DAMPs rather than that of HMGB1 alone, which has been implicated in COPD development [6], may be an important determinant of the susceptibility towards neutrophilic airway inflammation upon cigarette smoking.

In future studies it will be of interest to confirm whether a similar DAMP release signature is present in COPD patients, whether this is related to the susceptibility of smoking individuals to develop COPD and whether this signature can be used for the early detection of susceptibility to or the presence of COPD.

0 @ERSpublications

A specific profile of DAMPs identifies susceptibility towards smoke induced neutrophilic airway inflammation in mice http://ow.ly/tdgBL

Simon D. Pouwels ${ }^{1,2,3}$, Irene H. Heijink ${ }^{1,2,3}$, Antoon J.M. van Oosterhout ${ }^{1,2,4}$ and Martijn C. Nawijn ${ }^{1,2,4}$

${ }^{1}$ Dept of Pathology and Medical Biology, Laboratory of Allergology and Pulmonary Diseases, University of Groningen, University Medical Center Groningen, Groningen, and ${ }^{2}$ GRIAC Research Institute, University of Groningen, University Medical Center Groningen, Groningen, The Netherlands. ${ }^{3}$ Both authors contributed equally. ${ }^{4}$ Both authors contributed equally.

Correspondence: M.C. Nawijn, Laboratory of Allergology and Pulmonary Diseases, Dept of Pathology and Medical Biology, University Medical Center Groningen, IPC EA11, Hanzeplein 1, 9713GZ, Groningen, the Netherlands. E-mail: m.c.nawijn@umcg.nl

Received: July 252013 | Accepted after revision: Oct 292013 | First published online: Dec 52013

Support statement: This study was funded by the Netherlands Asthma Foundation (project 3.2.11.025), Stichting Astma Bestrijding (project 2013/008) and Top Institute Pharma (project T1-201).

Conflict of interest: None declared.

\section{References}

Halbert RJ, Natoli JL, Gano A, et al. Global burden of COPD: systematic review and meta-analysis. Eur Respir J 2006; 28: 523-532.

2 Brusselle GG, Joos GF, Bracke KR. New insights into the immunology of chronic obstructive pulmonary disease. Lancet 2011; 378: 1015-1026.

Kono H, Rock KL. How dying cells alert the immune system to danger. Nat Rev Immunol 2008; 8: 279-289.

Hacker S, Lambers C, Hoetzenecker K, et al. Elevated HSP27, HSP70 and HSP90 $\alpha$ in chronic obstructive pulmonary disease: markers for immune activation and tissue destruction. Clin Lab 2009; 55: 31-40.

5 Merkel D, Rist W, Seither P, et al. Proteomic study of human bronchoalveolar lavage fluids from smokers with chronic obstructive pulmonary disease by combining surface-enhanced laser desorption/ionization-mass spectrometry profiling with mass spectrometric protein identification. Proteomics 2005; 5: 2972-2980.

6 Ferhani N, Letuve S, Kozhich A, et al. Expression of high-mobility group box 1 and of receptor for advanced glycation end products in chronic obstructive pulmonary disease. Am J Respir Crit Care Med 2010; 181: 917-927. Castaldi PJ, Cho MH, Litonjua AA, et al. The association of genome-wide significant spirometric loci with chronic obstructive pulmonary disease susceptibility. Am J Respir Cell Mol Biol 2011; 45: 1147-1153.

8 Kaczmarek A, Vandenabeele P, Krysko DV. Necroptosis: the release of damage-associated molecular patterns and its physiological relevance. Immunity 2013; 38: 209-223.

9 Krysko DV, Garg AD, Kaczmarek A, et al. Immunogenic cell death and DAMPs in cancer therapy. Nat Rev Cancer 2012; 12: 860-875.

10 Paludan SR, Bowie AG. Immune sensing of DNA. Immunity 2013; 38: 870-880.

\section{Experimental rhinovirus 16 infection in moderate asthmatics on inhaled corticosteroids}

To the Editor:

The majority of asthma exacerbations are associated with respiratory virus infections, mostly rhinoviruses (RVs) [1], due to enhanced inflammation in the airways [2]. These occur despite symptom control with inhaled corticosteroids (ICS) [3]. Experimental RV infection is a valuable tool for studying virus-induced exacerbations $[2,4]$, but has, to date, involved only corticosteroid-nä̈ve asthmatics. We have, therefore, 